\title{
Self-Similar Solution of Heat and Mass Transfer of Unsteady Mixed Convection Flow on a Rotating Cone Embedded in a Porous Medium Saturated with a Rotating Fluid
}

\author{
Saleh M. Al-Harbi \\ Department of Mathematics, Makkah Teacher College, Makkah, Saudi Arabia \\ E-mail: salharbi434@yahoo.com \\ Received June 6, 2011; revised August 22, 2011; accepted August 29, 2011
}

\begin{abstract}
A self-similar solution of unsteady mixed convection flow on a rotating cone embedded in a porous medium saturated with a rotating fluid in the presence of the first and second orders resistances has been obtained. It has been shown that a self-similar solution is possible when the free stream angular velocity and the angular velocity of the cone vary inversely as a linear function of time. The system of ordinary differential equations governing the flow has been solved numerically using an implicit finite difference scheme in combination with the quasi-linearization technique. Both prescribe wall temperature and prescribed heat flux conditions are considered. Numerical results are reported for the skin friction coefficients, Nusselt number and Sherwood number. The effect of various parameters on the velocity, temperature and concentration profiles are also presented here.
\end{abstract}

Keywords: Unsteady Mixed Convection, Heat and Mass Transfer, Rotating Cone, Rotating Fluid, Porous Media, Self-Similar Solution

\section{Introduction}

The study and analysis of heat and mass transfer in porous media has been the subject of many investigations due to their frequent occurrence in industrial and technological applications. Examples of some applications include geothermal reservoirs, drying of porous solids, thermal insulation, enhanced oil recovery and many others. Cone-shaped bodies are often encountered in many engineering applications and the heat transfer problem of mixed convection boundary layer flow over a rotating cone, which occurs in rotating heat exchangers, are extensively used by the chemical and automobile industries. Moreover, convective heat on a rotating cone has several important applications such as design of canisters for nuclear waste disposal, nuclear reactor cooling system, geothermal reservoirs. Earlier investigations of flow and heat transfer in rotating systems are given by Hartnett and Deland [1], Hering and Grosh [2] and Tien and Tsuji [3]. Hering and Grosh [4] have obtained a number of similarity solutions for cones with prescribed wall tem- perature being a power function of the distance from the apex along the generator. Himasekhar et al. [5] found the similarity solution of the mixed convection flow over a vertical rotating cone in an ambient fluid for a wide range of Prandtl numbers. Wang [6] has also obtained a similarity solution of boundary layer flows on rotating cones, discs and axisymmetric bodies with concentrated heat sources. Further, Yih [7] has presented non-similar solutions to study the heat transfer characteristics in mixed convection about a cone in saturated porous media. The effect of thermal radiation on the non-Darcy natural convection flow over a vertical cone and wedge embedded in a porous medium with variable viscosity and wall mass flux was investigated numerically by EL-Harby [8]. All these studies pertain to steady flows.

There is a large body of literature on unsteady, boundary-layer flows past bodies of different geometries embedded in porous media. Takhar et al. [9] have presented a study on unsteady mixed convection flow over a vertical cone rotating in an ambient fluid with a time-dependent angular velocity in the presence of a magnetic 
field. Therefore, as a step towards the eventual development of studies on unsteady mixed convection flows, it is interesting as well as useful to investigate the combined effects of thermal and mass diffusion on a rotating cone in a rotating viscous fluid where the angular velocity of the cone and the free stream angular velocity vary arbitrarily with time. The problem of unsteady mixed convection flow on a rotating cone in a rotating fluid has been considered by Anilkumar and Roy [10]. The interested reader can find an excellent collection of papers on unsteady convective flow problems over heated bodies embedded in a fluid-saturated porous medium in the book papers by Pop and Ingham [11] and in the book by Nield and Bejan [12]. Hassanien et al. [13] have studied the unsteady free convection flow in the stagnation-point region of a rotating sphere embedded in a porous medium. Also, the problem of unsteady free convection flow in the stagnation-point region of a three-dimensional body embedded in a porous media has been studied by Hassanien et al. [14]. Recently, the problem of unsteady MHD free convection flow past a semi infinite vertical permeable moving plate with heat source and suction has been studied by Ibrahim et al. [15]. Roy et al. [16] have obtained Non-similar solution of an unsteady mixed convection flow over a vertical cone in the presence of surface mass transfer. The effect of combined viscous dissipation and Joule heating on unsteady mixed convention magnetohydrodynamics (MHD) flow on a rotating cone in an electrically conducting rotating fluid in the presence of Hall and ion-slip currents was investigated by Osalusi et al. [17].

The aim of the present paper is to develop a new self-similarity solutions for the heat and mass transfer of unsteady mixed convection flow on a rotating cone embedded in a porous medium saturated with a rotating fluid in the presence of the first and second orders resistances which to the best of our knowledge have not been investigated yet. The system of ordinary differential equations governing the flow has been solved numerically using the method of an implicit finite difference scheme.

\section{Mathematical Analysis}

We consider the unsteady laminar viscous incompressible fluid flowing over an infinite rotating cone in a rotating fluid-saturated porous medium. Both the cone and the fluid are rotating about the axis of the cone with time-dependent angular velocities either in the same direction or in the opposite direction. This introduces unsteadiness in the flow field. We have taken the rectangular co-ordinate system $(x, y, z)$ where $x$ is measured along a meridian section, the $y$-axis along a circular section and $z$-axis normal to the cone surface as shown in Figure 1. Let $u, v$ and $w$ be the velocity components along $x$ (tangential), $y$ (circumferential) and $z$ (normal) directions, respectively.

The buoyancy forces arise due to the temperature and concentration variations in the fluid and the flow is taken to be axi-symmetric. The wall and the free stream are maintained at a constant temperature and concentration. Under the above assumptions and using the Boussinesq approximation, the governing boundary layer momentum, energy and diffusion equation can be expressed as:

$$
\begin{gathered}
\frac{\partial(x u)}{\partial x}+\frac{\partial(x w)}{\partial z}=0 \\
\frac{\partial u}{\partial t}+u \frac{\partial u}{\partial x}+w \frac{\partial u}{\partial z}-\frac{v^{2}}{x}=-\frac{v_{e}^{2}}{x}+v \frac{\partial^{2} u}{\partial z^{2}} \\
-\frac{\varepsilon v}{k} u-\frac{\varepsilon^{2} \Gamma}{K^{1 / 2}} u^{2}+g \beta \cos \alpha^{*}\left(T-T_{\infty}\right) \\
+g \beta^{*} \cos \alpha^{*}\left(C-C_{\infty}\right), \\
\frac{\partial v}{\partial t}+u \frac{\partial v}{\partial x}+w \frac{\partial v}{\partial z}+\frac{u v}{x}=\frac{\partial v_{e}}{\partial t}+v \frac{\partial^{2} v}{\partial z^{2}} \\
\frac{\partial T}{\partial t}+u \frac{\partial T}{\partial x}+w \frac{\partial T}{\partial z}=\alpha \frac{\partial^{2} T}{\partial z^{2}} \\
\frac{\partial C}{\partial t}+u \frac{\partial C}{\partial x}+w \frac{\partial C}{\partial z}=D \frac{\partial^{2} C}{\partial z^{2}}
\end{gathered}
$$

The initial conditions are

$$
\begin{aligned}
& u(0, x, z)=u_{i}(x, z), \\
& v(0, x, z)=v_{i}(x, z), \\
& w(0, x, z)=u_{i}(x, z), \\
& T(0, x, z)=T_{i}(x, z), \\
& C(0, x, z)=C_{i}(x, z),
\end{aligned}
$$

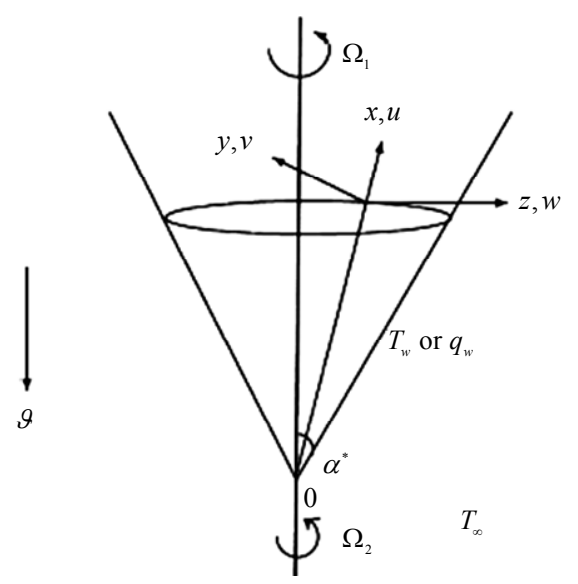

Figure 1. Physical model and coordinate system. 
and the boundary conditions are given by

$$
\begin{aligned}
& u(t, x, 0)=w(t, x, 0)=0, \\
& v(t, x, 0)=\Omega_{1} x \sin \alpha^{*}\left(1-s t^{*}\right)^{-1}, \\
& T(t, x, 0)=T_{w} \text { and } C(t, x, 0)=C_{w}, \\
& u(t, x, \infty)=0, \\
& v(t, x, \infty)=\Omega_{2} x \sin \alpha^{*}\left(1-s t^{*}\right)^{-1}, \\
& T(t, x, \infty)=T_{\infty} \text { and } C(t, x, \infty)=C_{\infty},
\end{aligned}
$$

here $\alpha^{*}$ is the semi-vertical angle of the cone; $v$ is the kinematics viscosity; $\rho$ is the density; $t$ and $t^{*}=\left(\Omega x \sin \alpha^{*}\right) t$ are the dimensional and dimensionless times, respectively; $\Omega_{1}$ and $\Omega_{2}$ are the angular velocities of the cone and the fluid far away from the surface, respectively; $\Omega\left(=\Omega_{1}+\Omega_{2}\right)$ is the composite angular velocity; $K$ and $\Gamma$ are the respective permeability and the inertia coefficient of the porous medium; $\varepsilon$ is the porosity; $\mathrm{g}$ is the acceleration due to gravity; $T$ is the temperature; $C$ is the species concentration; $\beta$ is the volumetric co-efficient of thermal expansion; $\beta^{*}$ is the volumetric co-efficient of expansion for concentration; $\alpha$ and $D$ are thermal and mass diffusivity, respectively; Subscripts $t, x$ and $z$ denote partial derivatives with respect to the corresponding variables and the subscripts $e$, $i, w$ and $\infty$ denote the conditions at the edge of the boundary layer, initial conditions, conditions at the wall and free stream conditions, respectively; $T_{0}, C_{0}, T_{\infty}$ and $C_{\infty}$ are constants.

Equations (1)-(5) are a system of partial differential equations with three independent variables $x, z$ and $t$. It has been found that these partial differential equations can be reduced to a system of ordinary differential equations, if we take the velocity at the edge of the boundary layer $v_{e}$ and the angular velocity of the cone to vary inversely as a linear function of time. Consequently, applying the following transformations:

$$
\begin{aligned}
& v_{e}=\Omega_{2} x \sin \alpha^{*}\left(1-s t^{*}\right)^{-1}, \\
& \eta=\left(\Omega \sin \alpha^{*} / v\right)^{1 / 2}\left(1-s t^{*}\right)^{-1 / 2} z, \\
& t^{*}=\left(\Omega \sin \alpha^{*}\right) t, \\
& u(t, x, z)=(-1 / 2) \Omega \sin \alpha^{*}\left(1-s t^{*}\right)^{-1} f^{\prime}(\eta), \\
& v(t, x, z)=\Omega x \sin \alpha^{*}\left(1-s t^{*}\right)^{-1} g(\eta), \\
& w(t, x, z)=\left(v \Omega \sin \alpha^{*}\right)^{1 / 2}\left(1-s t^{*}\right)^{-1 / 2} f(\eta),
\end{aligned}
$$

to Equations (1)-(5), we find that the continuity Equation (1) is identically satisfied, and Equations (2)-(5) reduce to,

$$
\begin{gathered}
f^{\prime \prime \prime}-f f^{\prime \prime}+\frac{1}{2} f^{2}-2\left[g^{2}-\left(1-\alpha_{1}\right)^{2}\right] \\
-2 \lambda_{1}(\theta+N \varphi)-s\left(f^{\prime}+\frac{1}{2} \eta f^{\prime \prime}\right) \\
+\gamma f^{\prime}-\Delta f^{\prime 2}=0, \\
g^{\prime \prime}-\left(f g^{\prime}-g f^{\prime}\right)+s\left(1-\alpha_{1}-g-\frac{1}{2} \eta g^{\prime}\right) \\
+\gamma g^{\prime}-\Delta g^{\prime 2}=0, \\
\operatorname{Pr}^{-1} \theta^{\prime \prime}-\left(f \theta^{\prime}-\frac{1}{2} f^{\prime} \theta\right)-s\left(2 \theta+\frac{1}{2} \eta \theta^{\prime}\right)=0, \\
S c^{-1} \varphi^{\prime \prime}-\left(f \varphi^{\prime}-\frac{1}{2} f^{\prime} \varphi\right)-s\left(2 \varphi+\frac{1}{2} \eta \varphi^{\prime}\right)=0,
\end{gathered}
$$

where $\gamma=\varepsilon /\left(D a_{L} \operatorname{Re}_{L}\right)$ is the first resistance parameter, $\Delta_{x}=2 \varepsilon^{2} \Gamma D a_{x}^{-1 / 2}$ is the second resistance parameter, $\operatorname{Re}_{L}=L^{2} \Omega \sin \alpha^{*} / v$ is the Reynolds number, $D a_{L}=K / L^{2}$ is the Darcy number, $\lambda_{1}=G r_{1} / \mathrm{Re}_{L}^{2}$, $\lambda_{2}=G r_{2} / \operatorname{Re}_{L}^{2}$ are the buoyancy parameters

$$
\begin{aligned}
& G r_{1}=\left(T_{0}-T_{\infty}\right) L^{3} g \beta \cos \alpha^{*} / v^{2}, \\
& G r_{2}=\left(T_{0}-T_{\infty}\right) L^{3} g \beta^{*} \cos \alpha^{*} / v^{2}
\end{aligned}
$$

are the Grashof numbers, $\alpha_{1}=\Omega_{1} / \Omega$ is the angular velocity of the cone to the composite angular velocity, $N=\lambda_{2} / \lambda_{1}$ is the ratio of Grashof numbers, $\operatorname{Pr}=v / \alpha$ is the Prandtl number and $S c=v / D$ is the Schmidt number. $s$ is the parameter characterizing the unsteadyness in the free stream velocity $v_{e}=\Omega_{2} x\left(1-s t^{*}\right)^{-1} \sin \alpha^{*}$. The flow is accelerating if $s>0$ provided $s t^{*}<1$ and the flow decelerating if $s<0$. Further, $\alpha_{1}=0$ implies that the cone is stationary and the fluid is rotating, $\alpha_{1}=1$ represents the case where the cone is rotating in an ambient fluid, and for $\alpha_{1}=0.5$, the cone and the fluid are rotating with equal angular velocity in the same direction. The ratio of Grashof numbers denoted by the parameter $N$ measures the relative importance of thermal diffusion in inducing the buoyancy forces which drive the flow. $N=0$ for no species diffusion, infinite for the thermal diffusion, positive for the case when the buoyancy forces due to temperature difference act in the same direction and negative when they act in the opposite direction.

The boundary conditions Equation (7) can be expressed as

$$
\begin{gathered}
f(0)=0=f^{\prime}(0), g(0)=\alpha_{1}, \theta(0)=\varphi(0)=1, \\
f^{\prime}(\infty)=0, g(\infty)=1-\alpha_{1}, \theta(\infty)=\varphi(\infty)=0 .
\end{gathered}
$$

Here $\eta$ is the similarity variable; $f$ is the dimensionless stream function; $f^{\prime}$ and $g$ are, the respectively dimensionless velocity along $x$ - and $y$-directions; $\theta$ and 
$\varphi$ are the dimensionless temperature and concentration.

The set of partial differential Equations (1)-(5) governing the flow has to be solved subjected to initial conditions (6) and boundary conditions (7). The ordinary differential Equations (9)-(12) under the boundary conditions (13) are solved using the self-similar solution which implies that the solution at different times may be reduced to a single solutions i.e., the solution at the one value of time $t$ is similar to the solution at any other value of time $t$. This similarity property permits a decrease in the number of independent variables from three to one (in the present case) and yields treatment using ordinary differential equations instead of partial differential equations.

The quantities of physical interest are as follows:

The surface skin friction co-efficient in $x$ - and $y$ - directions are, respectively, given by

$$
\begin{aligned}
C_{f x} & =2 \mu(\partial u / \partial z)_{z=0} /\left(\rho\left[\Omega x \sin \alpha^{*}\left(1-s t^{*}\right)^{-1}\right]^{-2}\right) \\
& =-\operatorname{Re}_{x}^{-1 / 2} f^{\prime \prime}(0), \\
C_{f y} & =2 \mu(\partial v / \partial z)_{z=0} /\left(\rho\left[\Omega x \sin \alpha^{*}\left(1-s t^{*}\right)^{-1}\right]^{-2}\right) \\
& =-\operatorname{Re}_{x}^{-1 / 2} g^{\prime}(0) .
\end{aligned}
$$

Thus

$$
\begin{aligned}
& C_{f x} \operatorname{Re}_{x}^{1 / 2}=-f^{\prime \prime}(0), \\
& C_{f y} \operatorname{Re}_{x}^{1 / 2}=-g^{\prime}(0),
\end{aligned}
$$

where $\operatorname{Re}_{x}=\Omega x^{2} \sin \alpha^{*}\left(1-s t^{*}\right)^{-1} / v$.

The Nusselt number and Sherwood number can be expressed as:

$$
\begin{aligned}
& N u \mathrm{Re}_{x}^{-1 / 2}=-\theta^{\prime}(0) \\
& S h \mathrm{Re}_{x}^{-1 / 2}=-\varphi^{\prime}(0),
\end{aligned}
$$

where

$$
N u=-k(\partial T / \partial z)_{z=0} /\left(T_{w}-T_{\infty}\right)
$$

and

$$
S h=-\rho D(\partial C / \partial z)_{z=0} /\left(C_{w}-C_{\infty}\right)
$$

\section{Results and Discussion}

The similarity Equations (9)-(12) are coupled nonlinear and exhibit no closed-form solution. Therefore, they must be solved numerically subject to the boundary conditions. The implicit finite-difference method with iteration similar to that discussed by Inouye and Tate [18] have proven to be successful for the solution of such equations and for this reason, it will be employed herein.

The computations have been carried out with $\Delta \eta=0.01$ for various values of $\operatorname{Pr}(7 \leq \operatorname{Pr} \leq 10), \quad \lambda(0 \leq \lambda \leq 5)$, $\alpha_{1}\left(-0.25 \leq \alpha_{1} \leq 1.0\right), \quad S c(0.22 \leq S c \leq 2.57), \quad s(-1 \leq s \leq 1)$, $N(-0.5 \leq N \leq 1.0), \gamma=0.0,0.5,1.0, \Delta=0.0,0.5,1.0$. The edge of the boundary layer $\eta_{\infty}$ is taken between 4 and 6 depending on the values of parameters. In order to verify the correctness of our method, we have compared our results with those of Himasekher et al. [5] and Anilkumar and Roy [10]. The results are found to be in excellent agreement and some of the comparisons are shown in Tables 1 and 2. Also the effects of $\lambda$ and $\alpha$ on the skin friction coefficients, Nusselt number and Sherwood number $\left(C_{f x} \operatorname{Re}_{x}^{1 / 2}, C_{f y} \operatorname{Re}_{x}^{1 / 2}, N u \mathrm{Re}_{x}^{-1 / 2}, S h \mathrm{Re}_{x}^{-1 / 2}\right)$ are presented in Table 3.

Figures 2-5 show the effect of the first resistance parameter $\gamma$, the second resistance parameter $\Delta$ and the buoyancy parameter $\lambda$ on the velocity profiles in the tangential direction $f^{\prime}(\eta)$, on the temperature profiles

\begin{tabular}{|c|c|c|c|c|}
\hline \multirow{2}{*}{$\operatorname{Pr}$} & \multirow{2}{*}{$\lambda$} & \multicolumn{3}{|c|}{ Present results } \\
\hline & & $-f^{\prime \prime}(0)$ & $-g^{\prime}(0)$ & $-\theta^{\prime}(0)$ \\
\hline \multirow{6}{*}{0.7} & \multirow{2}{*}{0.0} & 1.0189 & 0.6155 & 0.4347 \\
\hline & & $1.0256^{*}$ & $0.6158^{*}$ & $0.4299 *$ \\
\hline & \multirow{2}{*}{1.0} & 2.07736 & 0.84997 & 0.61173 \\
\hline & & $2.2021^{*}$ & $0.8496^{*}$ & $0.6120^{*}$ \\
\hline & \multirow{2}{*}{10} & 8.52447 & 1.40365 & 1.01729 \\
\hline & & $8.5041^{*}$ & $1.3990^{*}$ & $1.0097 *$ \\
\hline \multirow{2}{*}{1} & 0.0 & 1.0189 & 0.6155 & 0.51858 \\
\hline & 1.0 & 2.00333 & 0.82459 & 0.7003 \\
\hline & \multicolumn{4}{|c|}{ Anilkumar and Roy [10] } \\
\hline \multirow{3}{*}{0.7} & 0.0 & 1.0199 & 0.6160 & 0.4305 \\
\hline & 1.0 & 2.1757 & 0.8499 & 0.6127 \\
\hline & 10 & 8.5029 & 1.4061 & 1.0175 \\
\hline \multirow{2}{*}{1} & 0.0 & 1.0199 & 0.6160 & 0.51808 \\
\hline & 1.0 & 2.0627 & 0.8250 & 0.7005 \\
\hline
\end{tabular}
$\theta(\eta)$ and on the concentration profiles $\varphi(\eta)$ for accelerating flows $s=0.5, N=1.0, S c=0.94$ and $\operatorname{Pr}=0.7$. The presence of a porous medium in the flow presents resistance to flow, thus, slowing the flow and increasing the pressure drop across it.

Therefore, as the first and second resistances increases,

Table 1. Comparison of the results $\left(-f^{\prime \prime}(0),-g^{\prime}(0),-\theta^{\prime}(0)\right)$ with those of Himasekher et al. [5] and Anilkumar and Roy [10].

*Values taken from Himasekher et al. [5]. 
Table 2. Comparison of the results skin friction coefficients, Nusselt number and Sherwood number $\left(C_{f x} \operatorname{Re}_{x}^{1 / 2}, C_{f y} \operatorname{Re}_{x}^{1 / 2}\right.$, $N u \mathrm{Re}_{x}^{-1 / 2}$ ) when $N=1.0, \operatorname{Pr}=0.7, S c=0.94$ and $s=0.5$ with those of $(*)$ anilkumar and roy $[10]$.

\begin{tabular}{|c|c|c|c|c|c|}
\hline & $\alpha$ & $C_{f x} \operatorname{Re}_{x}^{1 / 2}$ & $C_{f y} \mathrm{Re}_{x}^{1 / 2}$ & $N u \mathrm{Re}_{x}^{-1 / 2}$ & $S h \mathrm{Re}_{x}^{-1 / 2}$ \\
\hline \multirow{11}{*}{1} & \multirow{2}{*}{-0.5} & -1.27349 & -1.33646 & 0.55691 & 0.66286 \\
\hline & & $-1.27215^{*}$ & $-1.33537 *$ & $0.55580^{*}$ & $0.66305^{*}$ \\
\hline & \multirow{2}{*}{0.0} & 0.63047 & -64157 & 0.82157 & 0.9514 \\
\hline & & $0.63241^{*}$ & $0.63949 *$ & $0.81922 *$ & $0.95065^{*}$ \\
\hline & \multirow{3}{*}{0.25} & 1.3139 & -0.2300 & 0.89215 & 1.02926 \\
\hline & & & & & \\
\hline & & $1.31339 *$ & $-0.22765^{*}$ & $0.89011 *$ & $1.02812 *$ \\
\hline & \multirow{2}{*}{0.5} & 1.84895 & 0.19508 & 0.93841 & 1.08038 \\
\hline & & $1.84798^{*}$ & $0.19806^{*}$ & $0.93700 *$ & $1.07977 *$ \\
\hline & \multirow{2}{*}{0.75} & 2.24483 & 0.62299 & 0.96586 & 1.11099 \\
\hline & & $2.24659 *$ & $0.62679 *$ & $0.96563 *$ & $1.11132 *$ \\
\hline \multirow{11}{*}{3} & \multirow{2}{*}{-0.5} & 2.43469 & -1.43508 & 0.91406 & 1.05979 \\
\hline & & $2.43934 *$ & $-1.43105 *$ & $0.91210^{*}$ & $1.05951 *$ \\
\hline & \multirow{2}{*}{0.0} & 3.79418 & -0.5989 & 1.0298 & 1.18687 \\
\hline & & $3.79522 *$ & $-0.59651^{*}$ & $1.02869 *$ & $1.18645^{*}$ \\
\hline & \multirow{3}{*}{0.25} & 4.31863 & -0.13985 & 1.06614 & 1.22666 \\
\hline & & & & & \\
\hline & & $4.31854 *$ & $-0.13691 *$ & $1.06539 *$ & $1.22639 *$ \\
\hline & \multirow{2}{*}{0.5} & 4.73919 & 0.33212 & 1.09142 & 1.25444 \\
\hline & & $4.73958 *$ & $0.33552 *$ & $1.09111 *$ & $1.25444^{*}$ \\
\hline & \multirow{2}{*}{0.75} & 5.05751 & 0.80937 & 1.10715 & 1.27191 \\
\hline & & $505951 *$ & $081201 *$ & $1.0712 *$ & $127223 *$ \\
\hline \multirow{12}{*}{5} & \multirow{3}{*}{-0.5} & 5.17896 & -1.55388 & 1.06599 & 1.23185 \\
\hline & & & & & \\
\hline & & $5.18154 *$ & $-1.55129 *$ & $1.06503 *$ & $1.23177 *$ \\
\hline & \multirow{2}{*}{0.0} & 6.36087 & -0.60918 & 1.14372 & 1.31644 \\
\hline & & $6.36147^{*}$ & $-0.60724^{*}$ & $1.14323^{*}$ & $1.31640 *$ \\
\hline & \multirow{3}{*}{0.25} & 6.82067 & -0.10787 & 1.16935 & 1.34436 \\
\hline & & & & & \\
\hline & & $6.82071^{*}$ & $-0.10547^{*}$ & $1.16887 *$ & $1.34416^{*}$ \\
\hline & \multirow{2}{*}{0.5} & 7.19184 & 0.40346 & 1.18759 & 1.36433 \\
\hline & & $7.19231 *$ & $0.40602 *$ & $1.18730 *$ & $1.36415^{*}$ \\
\hline & \multirow{2}{*}{0.75} & 7.47488 & 0.91921 & 1.19912 & 1.3771 \\
\hline & & $7.47647 *$ & $0.92102 *$ & $1.19926^{*}$ & $1.37740 *$ \\
\hline
\end{tabular}

Table 3. Skin friction coefficients, Nusselt number and Sherwood number $\left(C_{f x} \operatorname{Re}_{x}^{1 / 2}, C_{f y} \operatorname{Re}_{x}^{1 / 2}, N u \operatorname{Re}_{x}^{-1 / 2}, S h \operatorname{Re}_{x}^{-1 / 2}\right)$ for different values of the first resistance parameter $\gamma$ and second resistance parameter $\Delta$, when $N=1.0, \lambda=1, S c=$ 0.94 and $s=0.5, \alpha=0.5$.

\begin{tabular}{|c|c|c|c|c|c|}
\hline $\operatorname{Pr}$ & $\gamma$ & $C_{f x} \operatorname{Re}_{x}^{1 / 2}$ & $C_{f y} \mathrm{Re}_{x}^{1 / 2}$ & $N u \mathrm{Re}_{x}^{-1 / 2}$ & $\operatorname{Sh} \operatorname{Re}_{x}^{-1 / 2}$ \\
\hline \multirow{3}{*}{0.7} & 0.0 & 1.2545 & -0.30646 & 0.93274 & 1.05144 \\
\hline & 0.5 & 1.38577 & -0.40706 & 0.94519 & 1.06612 \\
\hline & 1.0 & 1.55139 & -0.52446 & 0.96054 & 1.08416 \\
\hline \multirow{3}{*}{3.0} & 0.0 & 1.04607 & -0.32232 & 1.75335 & 1.02897 \\
\hline & 0.5 & 1.15386 & -0.42986 & 1.7723 & 1.04142 \\
\hline & 1.0 & 1.29099 & -0.55603 & 1.79581 & 1.0569 \\
\hline \multirow{3}{*}{7.0} & 0.0 & 0.90501 & -0.33192 & 2.61364 & 1.01487 \\
\hline & 0.5 & 1.00123 & -0.44337 & 2.63338 & 1.02627 \\
\hline & 1.0 & 1.12502 & -0.5743 & 2.65829 & 1.04062 \\
\hline \multirow[t]{2}{*}{$\operatorname{Pr}$} & $\Delta$ & $C_{f x} \mathrm{Re}_{x}^{1 / 2}$ & $C_{f y} \mathrm{Re}_{x}^{1 / 2}$ & $N u \mathrm{Re}_{x}^{-1 / 2}$ & $\operatorname{Sh} \mathrm{Re}_{x}^{-1 / 2}$ \\
\hline & 0.0 & 1.2545 & -0.30646 & 0.93274 & 1.05144 \\
\hline \multirow[t]{3}{*}{0.7} & 0.5 & 1.27153 & -0.28184 & 0.93426 & 1.05325 \\
\hline & 1.0 & 1.29145 & -0.26046 & 0.93606 & 1.05538 \\
\hline & 0.0 & 1.04607 & -0.32232 & 1.75335 & 1.02897 \\
\hline \multirow[t]{3}{*}{3.0} & 0.5 & 1.05068 & -0.29681 & 1.75402 & 1.02933 \\
\hline & 1.0 & 1.05605 & -0.27486 & 1.75481 & 1.02976 \\
\hline & 0.0 & 0.90501 & -0.33192 & 2.61364 & 1.01487 \\
\hline \multirow[t]{2}{*}{7.0} & 0.5 & 0.90417 & -0.30572 & 2.61336 & 1.01457 \\
\hline & 1.0 & 0.90359 & -0.28324 & 2.61312 & 1.0143 \\
\hline
\end{tabular}

the resistance due to the porous medium increases and the velocity components decrease further as shown in Figures 2 and 3. Also, the effects of $\gamma, \Delta$ and $\lambda$ on the temperature profiles $\theta(\eta)$ and on the concentration profiles $\varphi(\eta)$ are shown in Figures $\mathbf{4}$ and 5. From these figures it is obvious that as the first and second resistances increases the temperature and concentration profiles decrease.

In Figures 6 and 7 the effects of the first resistance and the Prandtl number Pr on the temperature profiles $\theta(\eta)$ and the effects of the first resistance and the Schmidt number Sc on the concentration profiles $\varphi(\eta)$ for $s=0.5$, $N=1.0, S c=0.94$ are presented. Also, the effects of $\gamma, \Delta$ and $\operatorname{Pr}$ on the skin friction coefficients, Nusselt number and Sherwood number $\left(C_{f x} \operatorname{Re}_{x}^{1 / 2}, C_{f y} \operatorname{Re}_{x}^{1 / 2}\right.$, $\left.N u \mathrm{Re}_{x}^{-1 / 2}, S h \mathrm{Re}_{x}^{-1 / 2}\right)$ are given in Table 3. Figures 6 


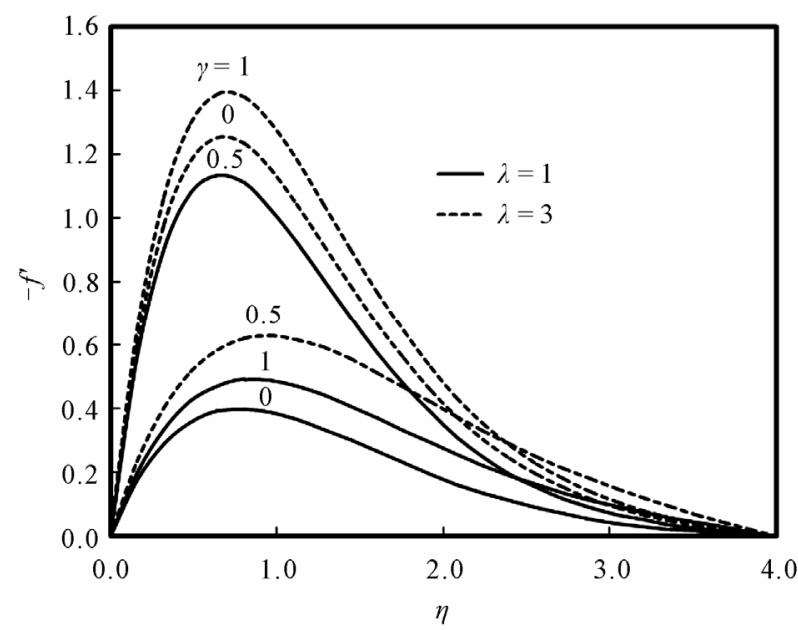

Figure 2. Effect of $\gamma$ and $\lambda$ on $-f^{\prime}$ for $\Delta=0.0, \alpha=0.25, S c=$ $0.94, s=0.5, N=1.0, \operatorname{Pr}=0.7$.

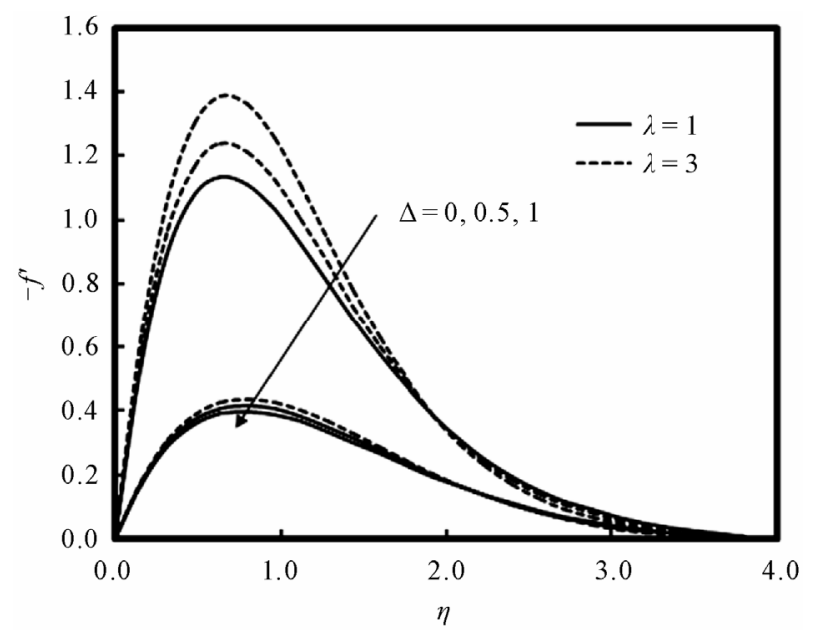

Figure 3. Effect of $\Delta$ and $\lambda$ on $-f^{\prime}$ for $\gamma=0.0, \alpha=0.25$. $S c=$ $0.94, s=0.5, N=1.0, \operatorname{Pr}=0.7$.

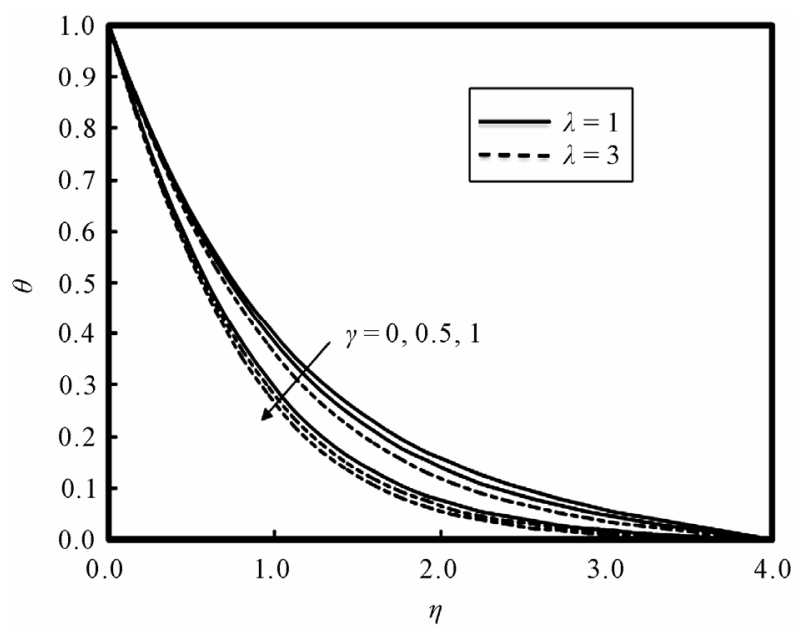

Figure 4. Effect of $\gamma$ and $\lambda$ on $\theta$ for for $\Delta=0.0, \alpha=0.25, S c=$ $0.94, s=0.5, N=1.0, \operatorname{Pr}=0.7$.

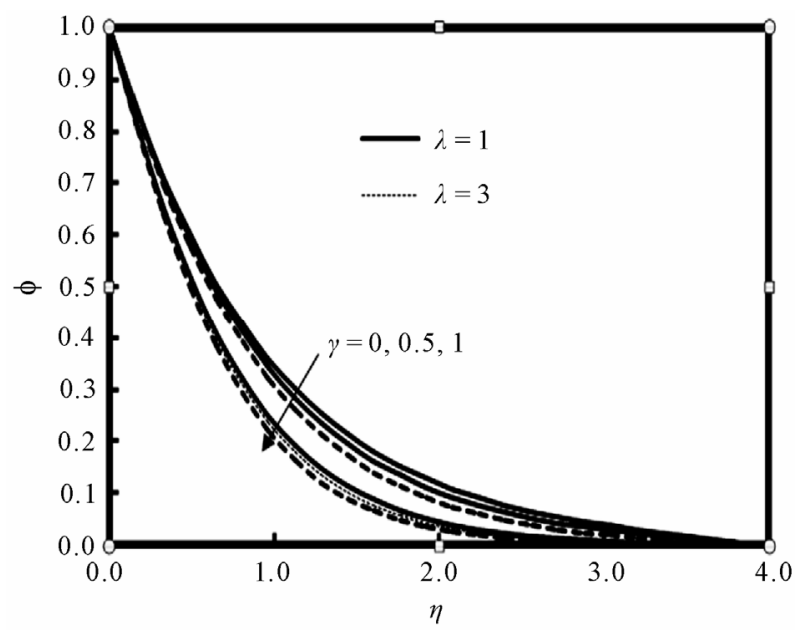

Figure 5. Effect of $\gamma$ and $\lambda$ on $\phi$ for $\Delta=0.0, \alpha=0.25, S c=$ $0.94, s=0.5, N=1.0, \operatorname{Pr}=0.7$.

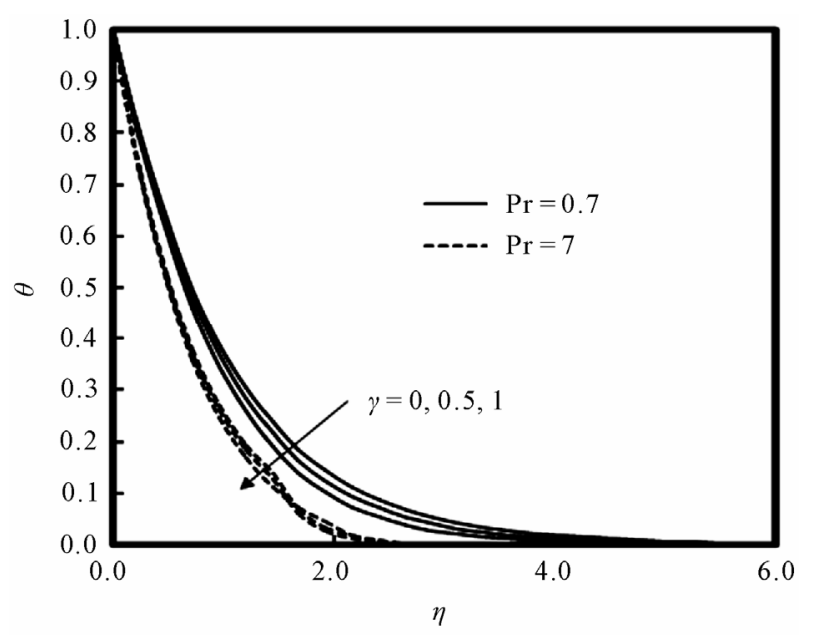

Figure 6. Effect of $\gamma$ and $\operatorname{Pr}$ on for $\gamma=0.0, \alpha=0.25 . S c=$ $0.94, s=0.5, N=1.0, \lambda=1.0$.

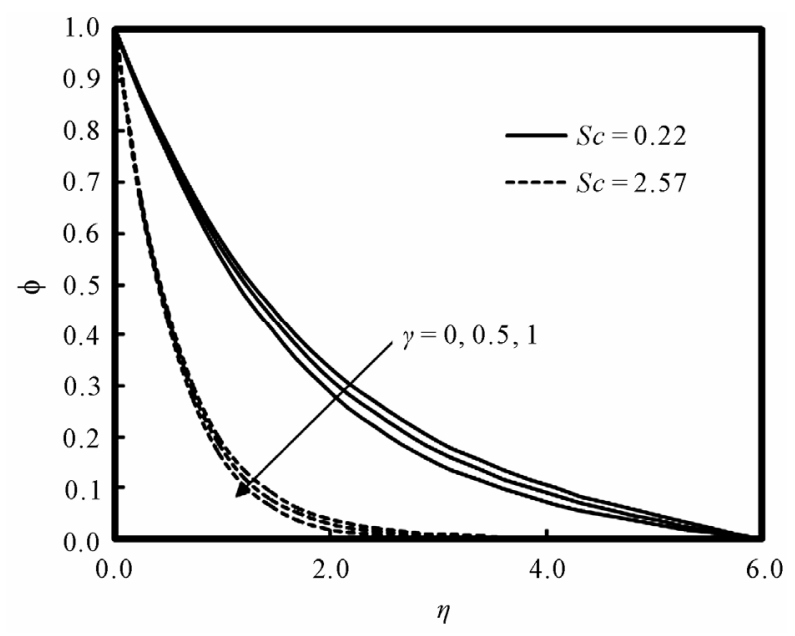

Figure 7. Effect of $\gamma$ and $S c$ on $\phi$ for $\Delta=0.0, \alpha=0.25, \lambda=$ 1.0, $s=0.5, N=1.0, \operatorname{Pr}=0.7$. 


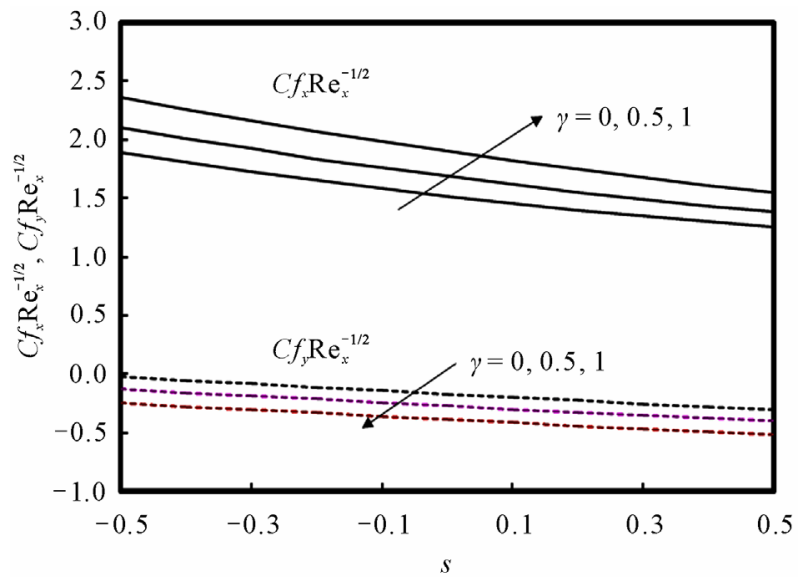

Figure 8. Effect of $\boldsymbol{\gamma}$ on Skin friction coefficients $\left(C f_{x} \mathrm{Re}_{x}^{-1 / 2}\right.$, $C f_{y} \operatorname{Re}_{x}^{-1 / 2}$ ) for $\Delta=0.0, \lambda=1.0, \alpha=0.25, S c=0.94, N=1.0$, $\operatorname{Pr}=0.7$.

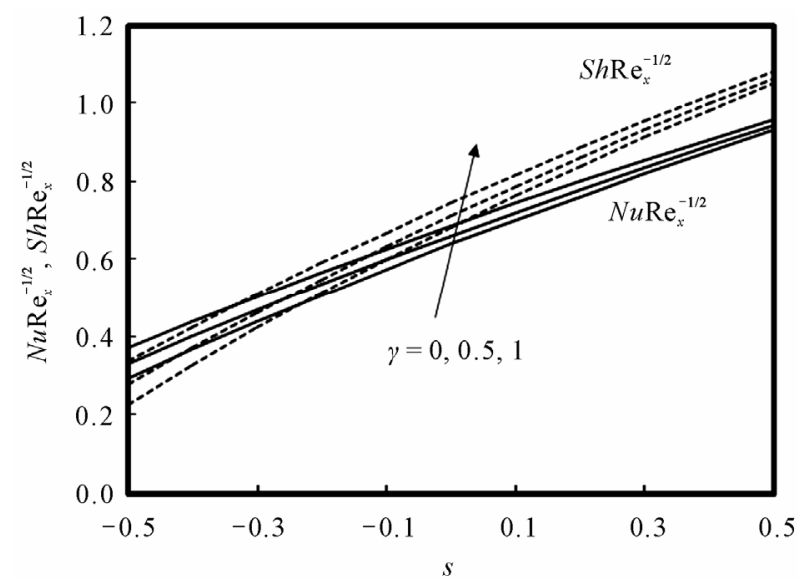

Figure 9. Effect of $\gamma$ on Nussellt and Sherood numbers $\left(N u \operatorname{Re}_{x}^{-1 / 2}, S h \operatorname{Re}_{x}^{-1 / 2}\right)$ for $\Delta=0.0, \lambda=1.0, \alpha=0.25, S c=0.94$, $N=1.0, \operatorname{Pr}=0.7$.

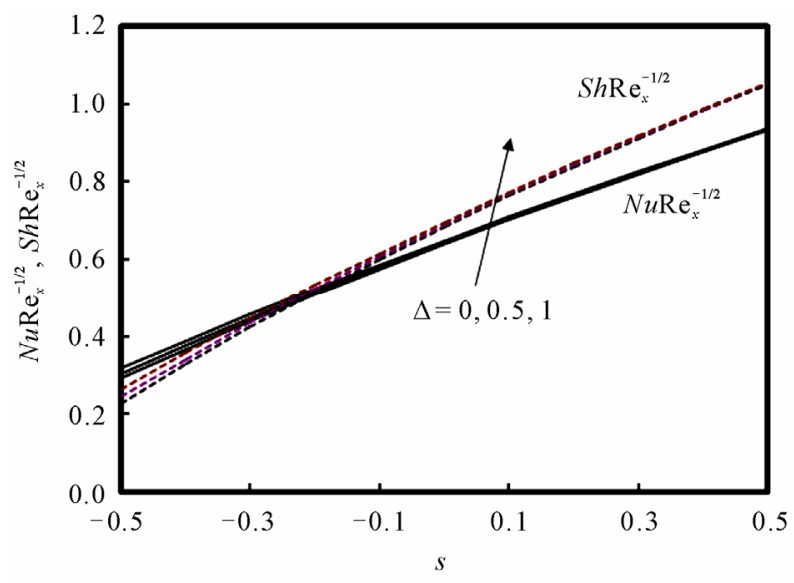

Figure 10. Effect of $\Delta$ on Nussellt and Sherood numbers $\left(N u \mathrm{Re}_{x}^{-1 / 2}, S h \mathrm{Re}_{x}^{-1 / 2}\right)$ for $\gamma=0.0, \lambda=1.0, \alpha=0.25, S c=0.94$, $N=$ 1.0, Pr $=0.7$.

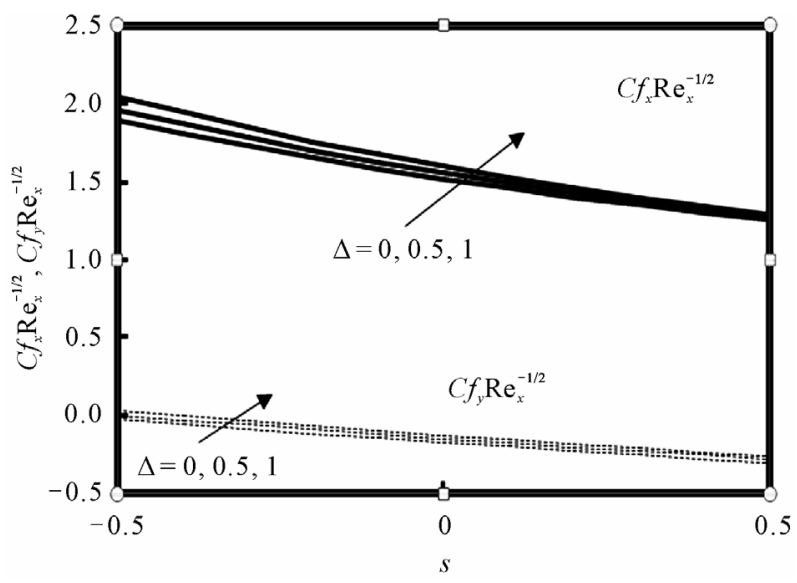

Figure 11. Effect of $\Delta$ on Skin friction coefficients $\left(C f_{x} \operatorname{Re}_{x}^{-1 / 2}\right.$, $\left.C f_{y} \operatorname{Re}_{x}^{-1 / 2}\right)$ for $\gamma=0.0, \lambda=1.0, \alpha=0.25, S c=0.94, N=1.0$, $\operatorname{Pr}=0.7$.

and 7 show that the increase in $\gamma, \operatorname{Pr}$ and $S c$ causes a reduction in thermal and concentration boundary layers, respectively. Hence in Table 3, Nu $\mathrm{Re}_{x}^{-1 / 2}$ increases with Pr and $S h \mathrm{Re}_{x}^{-1 / 2}$ increases with Sc.

Figures 8-11 display the effects of the first order resistance parameter, the second order resistance parameter and the unsteady parameter $S$ on the skin friction coefficients, Nusselt and Sherwood numbers $\left(C_{f x} \operatorname{Re}_{x}^{1 / 2}\right.$, $C_{f y} \mathrm{Re}_{x}^{1 / 2}, N u \mathrm{Re}_{x}^{-1 / 2}, S h \mathrm{Re}_{x}^{-1 / 2}$ ) for $\lambda=1.0, \alpha=0.25$, $S c=0.94$ and $\operatorname{Pr}=0.7$. From these figures it can be seen that as the unsteady parameter $S$ increases from -0.5 to 0.5 , the skin friction coefficients $\left(C_{f x} \operatorname{Re}_{x}^{1 / 2}, C_{f y} \mathrm{Re}_{x}^{1 / 2}\right)$ decrease, where the Nusselt and Sherwood numbers $\left(N u \mathrm{Re}_{x}^{-1 / 2}, S h \mathrm{Re}_{x}^{-1 / 2}\right)$ increase. Further, the same behavior is noticed for both the first and second order resistances parameter.

\section{References}

[1] J. P. Hartnett and E. C. Deland, "The Influence of Prandtl Number on the Heat Transfer from Rotating Non-Isothermal Disks and Cones," ASME Journal of Heat Transfer, Vol. 83, 1961, pp. 95-96.

[2] R. G. Hering and R. J. Grosh, "Laminar Free Convection from a Non-Isothermal Cone," International Journal of Heat and Mass Transfer, Vol. 5, No. 11, 1962, pp. 10591068. doi:10.1016/0017-9310(62)90059-5

[3] C. L. Tien and I. J. Tsuji, "A Theoretical Analysis of Laminar Forced Flow and Heat Transfer about a Rotating Cone," ASME Journal of Heat Transfer, Vol. 87, 1965, pp. 184-190.

[4] R. G. Hering and R. J. Grosh, "Laminar Free Convection from a Non-Isothermal Cone at Low Prandtl Number," International Journal of Heat and Mass Transfer, Vol. 8, No. 10, 1965. pp. 1333-1337. doi:10.1016/0017-9310(65)90059-1 
[5] K. Himasekhar, P. K. Sarma and K. Janardhan, "Laminar Mixed Convection from Vertical Rotating Cone," International Communications in Heat and Mass Transfer, Vol. 16, No. 1, 1989, pp. 99-106.

doi:10.1016/0735-1933(89)90045-6

[6] C. Y. Wang, "Boundary Layers on Rotating Cones, Discs and Axisymmetric Surfaces with a Concentrated Heat Source," Acta Mechanica, Vol. 81, No. 3-4, 1990, pp. 245-251. doi:10.1007/BF01176992

[7] K. A. Yih, "Mixed Convection about a Cone in a Porous Medium: The Entire Regime," International Communications in Heat and Mass Transfer, Vol. 26, No. 7, 1999, pp. 1041-1050. doi:10.1016/S0735-1933(99)00093-7

[8] S. M. Al-Harbi, "Numerical Study of Natural Convection Heat Transfer with Variable Viscosity and Thermal Radiation from a Cone and Wedge in Porous Media," Applied Mathematics and Computation, Vol. 170, 2005, pp. 64-75. doi:10.1016/j.amc.2004.10.093

[9] H. S. Takhar, A. Chamkha and G. Nath, "Unsteady Mixed Convention Flow from a Rotating Vertical Cone with a Magnetic Field," Heat and Mass Transfer, Vol. 39, No. 4, 2003, pp. 297-304.

[10] D. Anilkumar and S. Roy, "Unsteady Mixed Convection Flow on a Rotating Cone in a Rotating Fluid," Applied Mathematics and Computation, Vol. 155, No. 2, 2004, pp. 545-561. doi:10.1016/S0096-3003(03)00799-9

[11] I. Pop and D. B. Ingham, "Convective Heat Transfer: Mathematical and Computational Modeling of Viscous Fluids and Porous Media," Pergamon, Oxford, 2001.

[12] D. A. Nield and A. Bejan, "Convection in Porous Media," Springer-Verlag, New York, 2005.

[13] I. A. Hassanien, F. S. Ibrahim and Gh. M. Omer,
"Unsteady Free Convection Flow in the Stagnation-Point Region of a Rotating Sphere Embedded in a Porous Medium," Mechanical Engineering, Vol. 7, 2004, pp. 89-98.

[14] I. A. Hassanien, F. S. Ibrahim and Gh. M. Omer, "Unsteady Flow and Heat Transfer of a Viscous Fluid in the Stagnation Region of a Three-Dimensional Body Embedded in a Porous Medium," Journal of Porous Media, Vol. 9. No. 4, 2006, pp. 357-372. doi:10.1615/JPorMedia.v9.i4.60

[15] F. S. Ibrahim, A. M. Elaiw and A. A. Bakr, "Effect of Chemical Reaction and Radiation Absorption on the Unsteady MHD Free Convection Flow Past a Semi Infinite Vertical Permeable Moving Plate with Heat Source and Suction," Communications in Nonlinear Science and Numerical Simulation, Vol. 13, No. 6, 2008, pp. 10561066. doi:10.1016/j.cnsns.2006.09.007

[16] S. Roy, P. Datta and N. C. Mahanti, "Non-Similar Solution of an Unsteady Mixed Convection Flow over a Vertical Cone with Suction or Injection," International Journal of Heat and Mass Transfer, Vol. 50, 2007, pp. 181187. doi:10.1016/j.ijheatmasstransfer.2006.06.024

[17] E. Osalusi, J. Side, R. Harris and P. Clark, "The Effect of Combined Viscous Dissipation and Joule Heating on Unsteady Mixed Convection MHD Flow on a Rotating Cone in a Rotating Fluid with Variable Properties in the Presence of Hall and Ion-Slip Currents," International Communications in Heat and Mass Transfer, Vol. 35, No. 4, 2008, pp. 413-429. doi:10.1016/j.icheatmasstransfer.2007.09.002

[18] K. Inouye and A. Tate, "Finite Difference Version Quasilinearization Applied to Boundary Layer Equations," AIAA Journal, Vol. 12, No. 4, 1974, pp. 558-560. doi: $10.2514 / 3.49286$ 